\title{
TENDÊNCIAS DE MORTALIDADE POR DOENÇAS RESPIRATÓRIAS E DOENÇAS CARDIOVASCULARES EM TERESINA-PI
}

\author{
MORTALITY TRENDS DUE TO RESPIRATORY AND CARDIOVASCULAR DISEASES IN TERESINA, PIAUÍ, BRAZIL
}

TENDENCIAS DE MORTALIDAD POR ENFERMEDADES RESPIRATORIAS Y ENFERMEDADES CARDIOVASCULARES EN TERESINA, PIAUÍ,

BRASIL

Gleison Vitor Ferreira de Castro da Silva 1

Luciana Tolstenko Nogueira ${ }^{2}$

Izael Francisco de Brito Araujo ${ }^{3}$

Daiane Ribeiro de Souza ${ }^{4}$

Geovanna Arnaldo de Sousa ${ }^{5}$

Allan Dellon da Silva 6

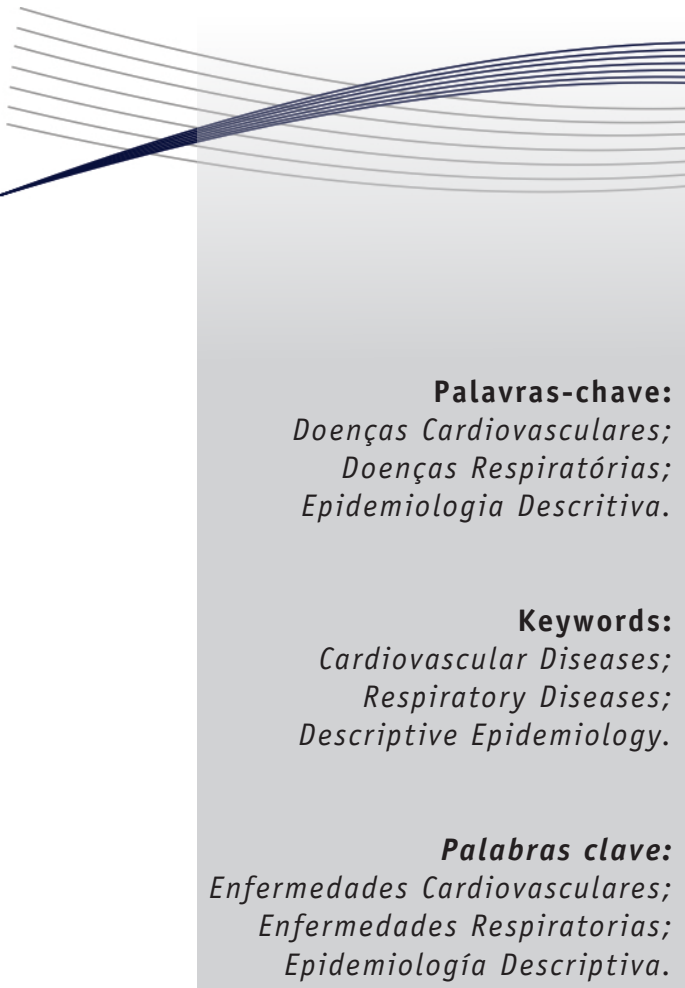

Submetido:

08/04/2020

Aprovado:

$12 / 05 / 2020$

Autor(a) para

Correspondência:

Gleison Vitor Ferreira de Castro

da Silva

R. Revanche, 2184, apto. 104

Aeroporto - Teresina (PI)

CEP: 64001-370

E-mail:gleisonvitorf@gmail.

\section{RESUMO}

Este estudo teve por objetivo analisar as tendencias de mortalidade por doenças respiratórias (DR) e doenças cardiovasculares (DCV) e comparar o comportamento dessas tendências no período entre 2009 e 2016, em TeresinaPI. Trata-se de estudo descritivo, com análise quantitativa, que teve como base os dados do Sistema de Informação sobre Mortalidade (SIM) e do Núcleo de Informação em Serviços de Saúde (NUINSA). O aumento proporcional de mortes por doenças do aparelho circulatório (DAC) foi um pouco maior que o de mortes por DR $(40,6 \%$ versus $38,6 \%)$, com destaque para o grupo de DAC para as doenças isquêmicas do coração (DIC) e as Doenças cerebrovasculares e no grupo de DR para a influenza e a pneumonia. 0 incremento nos óbitos por $D A C$ e DR entre 2009 e 2016, discretamente maior entre as primeiras, aponta a necessidade de comprometimento local com o plano de ações nacional para o enfrentamento das doenças crônicas não transmissiveis (DCNT) e, também, a fragilidade dos processos de saúde que visam à prevenção e ao cuidado.

1. Médico graduado pela Universidade Estadual do Piauí (UESPI).E-mail: gleisonvitorf@gmail.com ORCID: https:// orcid.org/0000-0003-4518-5136

2. Dentista. Professora na UESPI. Doutora em Ciências Odontológicas pela Faculdade São Leopoldo Mandic. E-mail: lutolstenko@hotmail.com ORCID: https://orcid.org/0000-0003-2547-7545

3. Médico graduado pela UESPI. E-mail: araujo.izael96@gmail.com ORCID: https://orcid.org/0000-0002-1248$\underline{2049}$

4. Médica graduada pela UESPI. E-mail: daianeribeirodesouza@gmail.com ORCID: https://orcid.org/0000-0003$\underline{2551-8300}$

4. Médica graduada pela UESPI. E-mail: geovannaarnaldo@gmail.com ORCID: https://orcid.org/0000-0003-18413690

5. Aluno de Graduação em Fisioterapia na UESPI. E-mail: dellonallan09@gmail.com ORCID: http://orcid.org/0000$\underline{0003-2137-5058}$

Certificação de redação científica: E.L.Freire Editora. Edição de texto: Evandro L. Freire. Preparação de original: Dida Bessana. 


\section{ABSTRACT}

This study aimed to analyze the mortality trends due to respiratory diseases (RDs) and cardiovascular diseases (CVDs) and to compare the behavior of these trends within the period from 2009 to 2016, in Teresina, Piaui, Brazil. This is a descriptive study, with a quantitative analysis, based on data from the Mortality Information System (Sistema de Informação sobre Mortalidade [SIM]) and the Health Services Information Center (Núcleo de Informação em Serviços de Saúde [NUINSA]). The proportional increased number of deaths due to circulatory system diseases (CSDS) was slightly higher than that of deaths due to RDs (40.6\% versus 38.6\%), highlighting the group of CSDs for ischemic heart diseases (IHDs) and cerebrovascular diseases and in the group of RDs for influenza and pneumonia. The increased number of deaths due to CSDs and RDs between 2009 and 2016, slightly higher among the first ones, points out the need for local commitment to the national action plan to tackle chronic noncommunicable diseases (CNCDs) and also the weak health processes aimed at prevention and care.

\section{RESUMEN}

Este estudio tuvo como objetivo analizar las tendencias de mortalidad por enfermedades respiratorias (ER) y enfermedades cardiovasculares (ECV) y comparar el comportamiento de estas tendencias dentro del periodo entre 2009 y 2016, en Teresina, Piauí, Brasil. Este es un estudio descriptivo, de análisis cuantitativo, que se basó en datos del Sistema de Información de Mortalidad (Sistema de Informação sobre Mortalidade [SIM]) y del Centro de Información de Servicios de Salud (Núcleo de Informação em Serviços de Saúde [NUINSA]). El aumento proporcional en las muertes por enfermedades del sistema circulatorio (ESC) fue ligeramente mayor que en las muertes por ER (40,6\% versus $38,6 \%)$, con énfasis en el grupo de las ESC para las enfermedades isquémicas del corazón (EIC) y las enfermedades cerebrovasculares y en el grupo de ER para influenza y neumonía. El aumento de las muertes por ESC y ER entre 2009 y 2016, un poco más alto entre los primeros, señala la necesidad de un compromiso local con el plan de acción nacional para combatir las enfermedades crónicas no transmisibles (ECNT) y, también, la fragilidad de los procesos de salud dirigidos a la prevención y a la atención.

\section{INTRODUÇÃO}

As doenças crônicas não transmissíveis (DCNT) levam a óbito cerca de 38 milhões de indivíduos a cada ano, sendo responsáveis por $70 \%$ das mortes no mundo ${ }^{1}$. No Brasil, as DCNT apresentam prioridade na área de saúde, pois o aumento de sua prevalência decorre do processo de mudança relativo à transição demográfica, epidemiológica e nutricional, visto que atualmente representam a principal causa de mortalidade no país ${ }^{2}$ Isso reforça o reconhecimento da importância da estruturação da vigilância epidemiológica das DCNT nos níveis de gestão municipal e estadual como uma importante ferramenta para seu enfrentamento e controle ${ }^{3}$.

A alta carga de mortalidade das DCNT pode ser verificada nos países de baixa e média renda, sendo os principais grupos: a) doenças do aparelho circulatório $(D A C)$; b) doenças respiratórias crônicas $(D R C) ; c)$ neoplasias; e d) diabetes ${ }^{4}$.

Segundo dados da Organização Mundial da Saúde (OMS), a estimativa é de que anualmente 17,9 milhões de pessoas morrem devido às doenças cardiovasculares (DCV), representando $44 \%$ de todas as mortes por $D_{C N T^{5}}$. Logo, as DCV constituem a principal causa de morte no mundo ${ }^{5-6}$.

As DAC estão entre as causas de óbito mais frequentes e as estimativas mostram que representam em torno de $31 \%$ de todos os óbitos no Brasil'. Em 2015, dois subgrupos dessas enfermidades (doenças isquêmicas do coração [DIC] e Doenças cerebrovasculares) foram as principais causas de morte no país, apesar de seu padrão de declínio verificado nos anos de 1996 a 2007, principalmente entre as Doenças cerebrovasculares (34\%) e outras formas de doenças cardíacas (44\%). No entanto, há situações epidemiológicas contrastantes entre as diferentes regiões do país, visto que no Nordeste houve aumento nas taxas de mortalidade por DIC e Doenças cerebrovasculares não observadas nas demais 9 .

No Brasil, em 2017, foi estimado um número de 383.961 mortes por DCV. Projeções da Sociedade Brasileira de Cardiologia ( $S B C$ ) apontam que, no fim de 2020, quase 400 mil cidadãos brasileiros morrerão por doenças do coração e da circulação ${ }^{10}$. 
As doenças respiratórias (DR) são caracterizadas como infecções que obstruem a passagem de ar tanto no nível do trato respiratório superior quanto no inferior e estão entre as infecções com maior índice de morbimortalidade do mundo ${ }^{11}$, o que pode ser comprovado pela evolução do panorama das DR no mundo, corroborando os crescentes desafios aos sistemas de saúde, relacionados a uma mudança de paradigma epidemiológico ${ }^{12}$.

A preocupação em conhecer a frequência da mortalidade por DR e por DCV data de muitos anos e a importância dos registros de mortalidade por essas causas básicas tem grande valor para a política de saúde pública regional. Assim, surgiu a necessidade de uma pesquisa usando o Sistema de Informação sobre Mortalidade (SIM), pois, ao avaliar as tendências de crescimento da mortalidade nesses dois grandes grupos de doenças, será possível descrever se realmente houve aumento de mortalidade por ambos os grupos de maneira homogênea ou se o grupo de mortalidade por DR apresentou crescimento proporcionalmente superior ao grupo de mortalidade por DCV, ou o inverso.

Em tal contexto, este estudo teve por objetivo analisar as tendências de mortalidade por doenças respiratórias e doenças cardiovasculares e comparar o comportamento dessas tendências entre 2009 a 2016, em Teresina-PI.

\section{METODOLOGIA}

Trata-se de estudo descritivo, de análise quantitativa, conduzido em Teresina, capital do Piauí, estado situado na Região Nordeste do Brasil, com população estimada em 841.600 habitantes ${ }^{13}$.

A pesquisa teve como base fundamental os dados do Sistema de Informação sobre Mortalidade (SIM), tendo por objeto os dados quantitativos sobre causa básica de morte por DCV (CID-10 I00 a I99) e por DR (CID-10 J00 a J99). 0s dados foram coletados em 2017, com autorização, apoio e supervisão de profissionais do Núcleo de Informação em Serviços de Saúde (NUINSA), vinculado à Fundação Municipal de Saúde, e referem-se aos anos de 2009 a 2016, de todos os residentes em Teresina falecidos por causa de DCV e DR. Com base nesses dados foi possivel descrever o perfil de crescimento proporcional entre esses dois grandes grupos de doenças, bem como avaliar como está ocorrendo, nesse município, o processo de mudança do perfil epidemiológico de mortalidade pelas causas básicas já citadas.

\section{... a importância dos registros de mortalidade por essas causas básicas tem grande valor para a política de saúde pública...}

A análise de tendência dos coeficientes de crescimento da mortalidade se deu por meio de modelos de regressão linear simples. A construção de diagramas de dispersão dos óbitos e anos de estudo permite mostrar se ocorreu alguma suposição de evolução linear, para que se possa usar modelos lineares.

Este estudo seguiu os critérios propostos na Resolução n. 466/2012, do Conselho Nacional de Saúde (CNS), do Ministério da Saúde (MS ${ }^{14}$, e obteve aprovação do Comitê de Ética em Pesquisa da Universidade Estadual do Piauí (CEP UESPI), sob o Parecer n. 1.643.877/2016. Respeitou-se a dignidade humana e garantiu-se a devida proteção aos participantes da pesquisa, os quais, durante a coleta de dados, comprometeram-se a manter a confidencialidade sobre os dados coletados.

\section{RESULTADOS E DISCUSSÃO}

Ao comparar as taxas de mortalidade por $100 \mathrm{mil}$ habitantes por DCV e DR em Teresina, entre 2009 e 2016, observou-se um aumento proporcionalmente maior entre as DCV: a taxa de mortalidade foi de 155/100 mil habitantes em 2009 e saltou para 218/100 mil habitantes em 2016, representando um incremento de $63(40,6 \%)$, ao passo que a taxa de mortalidade por DR foi de $44 / 100$ mil habitantes em 2009 para 61/100 mil habitantes, em 2016, um aumento de $17(38,6 \%)$.

No entanto, o comportamento dessas taxas de mortalidade, tanto por DCV quanto por DR, ao longo dos anos, não registrou crescimento linear, como exemplificado na Figura 1, havendo anos em que as taxas decresceram em comparação ao ano anterior. No caso das DCV, houve queda nas taxas de mortalidade em comparação ao ano anterior nos anos de 2010 (-33 ou -21,3\%), 2012 (-12 ou $-6,7 \%)$, 2013 ( -17 ou $-10,2 \%)$ e 2015 ( -2 ou $-1,1 \%)$, ao passo que houve incremento nessas taxas de mortalidade nos anos de $2011(+56$ ou $+45,9 \%), 2014$ (+28 ou $+18,8 \%)$ e 2016 (+43 ou $+24,6 \%)$. Ao observar o 
comportamento das taxas de mortalidade por DR em comparação ao ano anterior, nota-se aumento nos anos de $2011(+14$ ou $+34,1 \%), 2013(+10$ ou $+19,2 \%)$ e $2015(+9$ ou $+14,7 \%)$ e redução nos anos de $2010(-3$ ou $-6,8 \%)$, $2012(-3$ ou $-5,4 \%) 2014(-1$ ou $-1,6 \%)$ e 2016 (-9 ou $-12,8 \%)$.

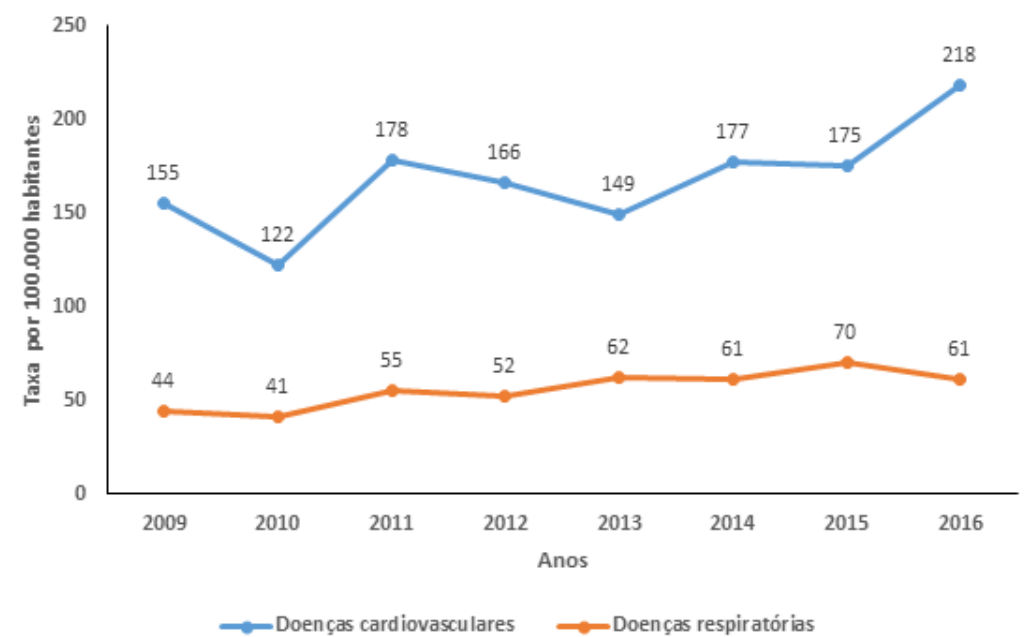

Figura 1 - Série histórica das taxas de mortalidade por doenças cardiovasculares e doenças respiratórias (100 mil habitantes) na população de Teresina (2009-2016).

Fonte: Elaborada pelos autores.

Como mostra a Figura 2, no grupo de DCV, as 6 principais causas de óbito encontradas foram as DIC (CID 10: I20 a I25), com mediana do número de óbitos de 260 entre 2009 e 2016, seguidas pelas Doenças cerebrovasculares (CID 10: I60 a I69), com mediana de 150, doenças hipertensivas (DH) (CID 10: I10 a I15), com mediana de 107, outras formas de doença do coração (CID 10: I30 a I52), com mediana de 27, doença cardíaca pulmonar e doença da circulação (CID 10: I26 a I25), com mediana de 8, e doenças das veias, dos vasos linfáticos e dos gânglios linfáticos (CID 10: I80 a I89), com mediana de 6. 0s demais grupos de causas de óbito entre as DCV (doenças das artérias, arteríolas e capilares, doenças reumáticas crônicas do coração, febre reumática aguda e outras) tiveram mediana de óbitos no período avaliado inferior ou igual a 5.

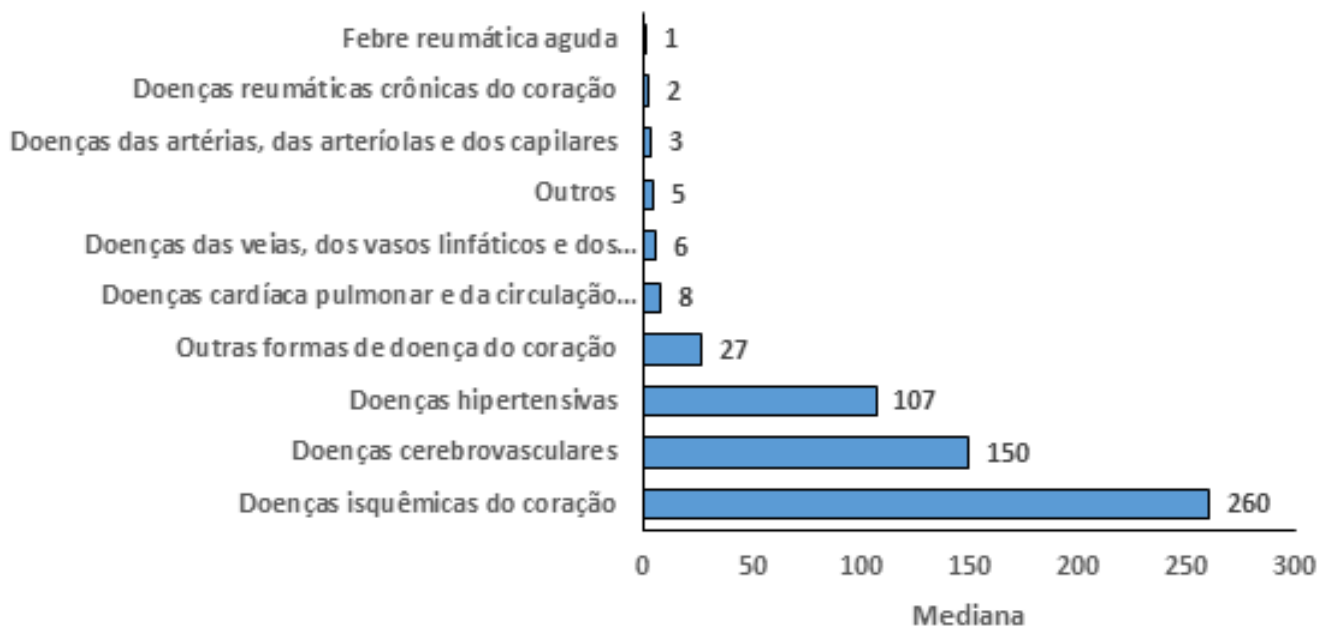

Figura 2 - Mediana do número de óbitos por doenças cardiovasculares na população de Teresina (2009-2016).

Fonte: Elaborada pelos autores. 
Já entre as DR (Figura 3), as 6 principais causas de óbito foram: a) influenza (gripe) e pneumonia (CID 10: J09 a J18), com mediana de 193; b) outras infecções agudas das vias aéreas inferiores (VAI) (CID 10: J20 a J22), com mediana de 42; c) doenças crônicas das VAI (CID 10: J40 a J47), com mediana de 17; d) doenças pulmonares devido a agentes externos (CID 10: J60 a J70), com mediana de 17; e) outras DR que afetam o interstício (CID 10: J80 a J84), com mediana de 7; e f) outras (CID 10: J95 a J99), com mediana de 7. 0s outros grupos de causas de óbito entre as DR (afecções necróticas e supurativas das VAI, outras doenças da pleura, infecções agudas das vias aéreas superiores [VAS], outras doenças das VAS e outras infecções agudas das VAS) tiveram mediana de óbitos menor ou igual a 4.

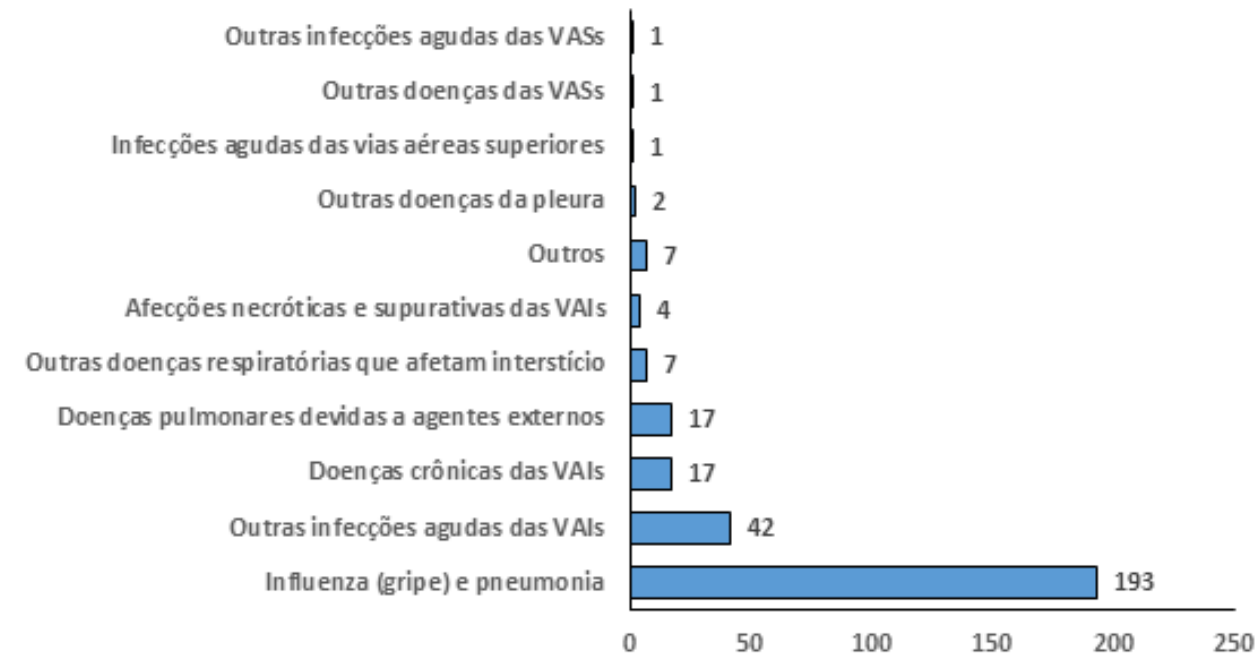

Figura 3 - Mediana do número de óbitos por doenças respiratórias na população de Teresina (2009-2016).

Fonte: Elaborada pelos autores.

Nas tabelas 1 e 2 constam os valores absolutos e relativos do número de óbitos por grupo de causas entre 2009 a 2016, entre as DCV e DR, respectivamente, podendo-se observar o comportamento desses valores ao longo dos anos, seja de forma decrescente ou crescente.

Em relação aos óbitos ocorridos por DCV nos anos de 2009 a 2016, em Teresina, foi possível descrever que as Doenças cerebrovasculares ( 4.243 casos), DIC (2.580 casos) e DH (2.163 casos) foram as responsáveis pelo maior número de óbitos das DCV, conforme apresentado na Tabela 1. 


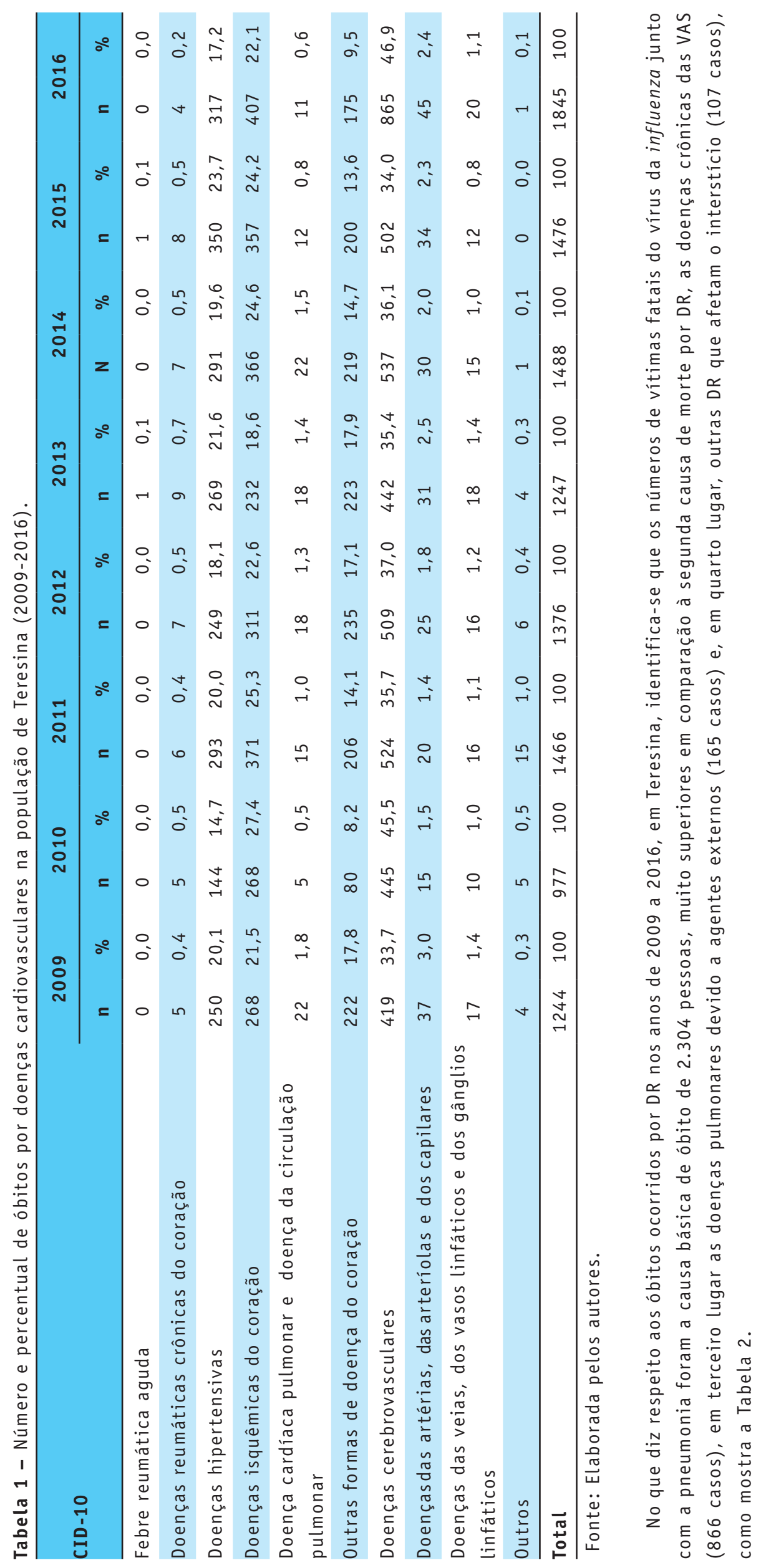

12 - SANARE (Sobral, Online). 2020 Jan-Jun;19(1):07-17 


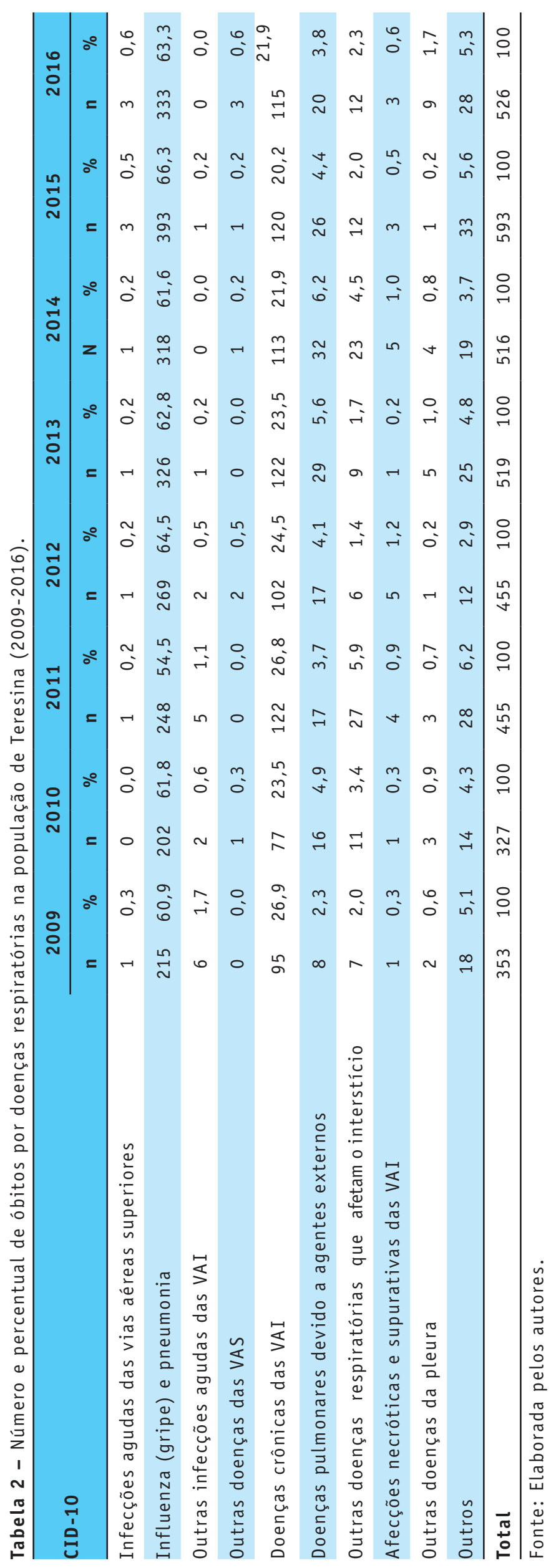


0 envelhecimento da população brasileira apresentou grande impacto social, econômico e epidemiológico no país e os declínios conjuntos das taxas de mortalidade e fecundidade ao longo do tempo foram determinantes no envelhecimento populacional, com ganho de anos de vida em todas as faixas etárias. A melhoria da qualidade de vida, o aumento da escolaridade, as opções de estilo de vida mais saudável, além da maior cobertura das ações de saúde pública e assistência médica têm contribuído para a maior esperança de vida e para eliminar parte dos óbitos precoces.

A queda nas taxas de mortalidade por DCNT verificada no Brasil, em especial nas taxas de DCV e DR, pode ser atribuída, parcialmente, à grande expansão do acesso aos serviços de saúde, ao declínio do tabagismo, bem como à melhora nas condições socioeconômicas da população de forma geral ${ }^{15-16}$.

Em contrapartida, a Região Nordeste, área de enorme concentração de renda, mas com evidente desigualdade social, quando comparada às demais regiões brasileiras apresenta um contínuo crescimento da taxa de mortalidade das DCNT devido aos fatores de risco serem mais prevalentes na região, conforme estudo da $S B C^{9,17}$.

Esse estudo descreveu a mortalidade por DR e DCV em Teresina, no período de 2009 a 2016. Entre os principais resultados se destacam 0 aumento de $40,6 \%$ da incidência de DCV e de $38,6 \%$ em DR, corroborando as tendências apresentadas na região de origem do local de pesquisa. Essa discrepância pode ser atribuída a características específicas das regiões, que têm perfil epidemiológico e de acesso aos serviços de saúde distinto, tanto em termos de características geográficas quanto da organização do serviço de saúde 9 .

A Teoria de $0 \mathrm{mram}^{18}$ afirma que a humanidade teria atravessado 3 fases epidemiológicas ao longo de sua história: a) a Era da Fome e das Pestilências, do início dos tempos históricos até o fim da Idade Média; b) a Era do Declínio das Pandemias, da Renascença ao início da Revolução Industrial; e c) a Era das Doenças Degenerativas e das Causadas pelo Homem, da Revolução Industrial até os tempos modernos. 0 quarto estágio da transição epidemiológica seria a Era do Retardamento das Doenças Degenerativas, surgida recentemente em razão do rápido aumento das populações idosas. Todavia, essa nova fase constitui um desafio para os grandes sistemas de saúde e de seguridade social ${ }^{19}$.

Estudos apontam, conforme dados de oscilações

\section{No Brasil, como nos outros países, as DCNT constituem o problema de saúde de maior magnitude...}

das taxas de mortalidade das DCV e DR, que as regiões Sul e Sudeste já iniciaram a quarta era da transição epidemiológica, ao passo que o Norte e o Nordeste ainda têm características marcantes dos estágios anteriores ${ }^{9}$.

A SBC aponta que na Região Nordeste foi encontrado o maior percentual (32\%) de indivíduos com pressão arterial média acima de $140 / 90 \mathrm{mmHg}$, além do maior percentual de indivíduos de 45 a 54 anos com colesterol elevado, maior percentual de indivíduos com triglicérides elevados e alto percentual (91\%) de indivíduos que não praticavam nenhuma atividade física ${ }^{17}$. Estes se configuram como os principais fatores de risco modificáveis para as DIC (260 óbitos no período estudado), DCV (150 óbitos no período estudado) e DH (107 óbitos no período estudado) que compartilham a aterosclerose como causa principal. Sendo esta um processo difuso e de progressão lenta, que pode ter seu início na infância, culminando com o aparecimento de DIC sintomáticas, geralmente na vida adulta e, sobretudo, em idosos ${ }^{17-20}$.

Instituído em 2011, atualmente está em vigência um plano de ações nacional de enfrentamento da DCNT, preconizado pelo MS, o qual define e prioriza as ações e os investimentos necessários, metas e compromissos a serem assumidos pelo Brasil, a fim de preparar o país para desafios das DCNT e seus fatores de risco nos próximos 10 anos. Seus objetivos levam em conta as metas globais para a redução das taxas de mortalidade por DCNT: redução de fatores de risco, como tabaco, álcool, sal e obesidade ${ }^{21}$. Além disso, o plano inclui aumento dos níveis de atividade física, do consumo de frutas e verduras e das coberturas do exame, que não constam como metas globais ${ }^{9}$.

Dados obtidos neste estudo avaliando o período 2009 a 2016 em Teresina evidenciam aumento de $38,6 \%$ da taxa de mortalidade por DR. No Brasil, como nos outros países, as DCNT constituem o problema de saúde de maior magnitude, responsáveis por $72 \%$ das causas de óbito; já a DRC corresponde a 5,8\% dessa 
mortalidade 7 , podendo atingir indivíduos de todas as esferas sociais ${ }^{22}$, embora se mostre mais intensa em indivíduos pertencentes a grupos vulneráveis, como os idosos e os de baixa escolaridade e renda ${ }^{23}$.

Além disso, dados deste estudo evidenciaram uma taxa de mortalidade por influenza e pneumonia variando de $60,9 \%$ a $63,3 \%$ como as principais causas de mortalidade por DR, entre 2009 e 2016. Considera-se fundamental a imunização anual contra a gripe de grupos prioritários. No entanto, apesar de fundamental a imunização anual contra a gripe de grupos prioritários, a prevalência dessa vacinação nas populações adulta e idosa com DRC tem-se mostrado abaixo da meta estabelecida pelo MS. Ainda assim, segundo um estudo anterior, observouse maior prevalência de vacinação para os idosos no Sul em comparação aos do Nordeste e, entre as doenças pulmonares citadas, a bronquite crônica foi a mais referida ${ }^{24}$.

\section{CONCLUSÃO}

Este estudo permitiu concluir que houve significativo aumento nas taxas de mortalidade tanto por DCV quanto por DR, entre 2009 a 2016, mostrando que as políticas públicas de saúde ainda são insuficientes para seguir o plano de ações nacional para enfrentamento das DCNT. Há, ainda, uma importante fragilidade no que respeita à conscientização da população geral acerca dos fatores de risco para o surgimento de doenças do aparelho circulatório e DR, preparo dos profissionais de saúde, além de acesso a serviços de saúde de qualidade.

Com esta pesquisa, observou-se a grande importância do sistema de informação em saúde, especialmente o SIM, para o fornecimento de dados os mais acurados possiveis para o planejamento e a gestão de políticas públicas voltadas à melhoria da efetividade no tratamento e no controle das DR e do aparelho circulatório.

Sugere-se a realização de futuros estudos como este para traçar o perfil e o padrão de crescimento ou decrescimento das doenças descritas, pois se espera que, com a melhoria das políticas públicas no manejo, no controle e no tratamento dessas doenças, seja possível diminuir seu padrão de crescimento em Teresina e em outras localidades do Brasil.

Este estudo teve como limitações possiveis subnotificações de mortes tanto por doenças do aparelho circulatório quanto por DR, pois o

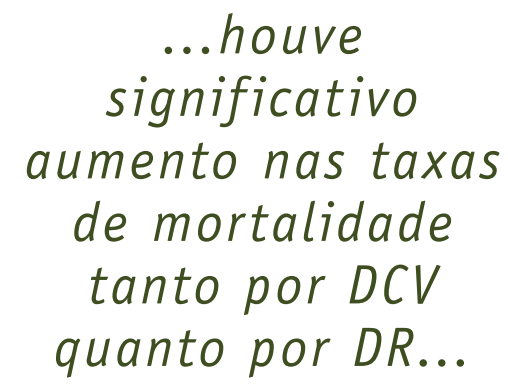

desenho do estudo proposto consistiu em analisar as tendências de crescimento dessas 2 categorias de doenças nos dados do SIM do Município de Teresina. Contudo, caso tenha havido mortes por essas 2 categorias de doenças não notificadas no SIM, elas não puderam ser inseridas neste estudo, devido ao desenho de pesquisa adotado.

\section{CONTRIBUIÇÃO DOS AUTORES}

Gleison Vitor Ferreira de Castro da Silva, Izael Francisco de Brito Araujo, Daiane Ribeiro de Souza e Geovanna Arnaldo de Sousa contribuíram com a realização da pesquisa, o delineamento do estudo e a redação do manuscrito. Luciana Tolstenko Nogueira contribuiu com o delineamento do estudo e a redação e revisão crítica do manuscrito. Allan Dellon da Silva contribuiu com a redação do manuscrito.

\section{REFERÊNCIAS}

1. World Health Organization. Health statistics and information systems: disease burden and mortality estimates. Geneva: WHO; 2016.

2. Miranda GMD, Mendes ACG, Silva ALA. 0 envelhecimento populacional brasileiro: desafios e consequências sociais atuais e futuras. Rev Bras Geriatr Gerontol [serial on the internet]. 2016 [cited 2020 May 11];19(3):507-19. Available from: https:// www.scielo.br/pdf/rbgg/v19n3/pt 1809-9823rbgg-19-03-00507.pdf

3. Brasil. Panorama da vigilância de doenças crônicas não transmissíveis no Brasil, 2018. Bol Epidemiol [serial on the internet]. 2019 [cited 2020 May 11];50(40). Available from: https://www. saude.gov.br/images/pdf/2020/janeiro/03/Boletimepidemiologico-SVS-40.pdf

4. Alves CG, Morais Neto 0L. Tendência da mortalidade prematura por doenças crônicas não transmissiveis nas unidades federadas brasileiras. Ciênc Saúde Colet [serial on the internet]. 2015 [cited 2020 May 11];20(3):641-54. Available from: https://www.scielo.br/pdf/csc/v20n3/pt 14138123-csc-20-03-00641.pdf 
5. World Health Organization. World health statistics 2018: monitoring health for the SDGs, sustainable development goals. Geneva: WHO; 2018.

6. Lima JBD. CADE-Q SV: prático e relevante na avaliação dos pacientes com doenças cardiovasculares sobre a sua condição de saúde. Arq Bras Cardiol [serial on the internet]. 2018 [cited 2020 May 11];111(6):850-1. Available from: https://www.scielo.br/scielo.php?script=sci arttext\&pid $=$ S0066-782X2018001800850\&lng =pt\&tl $\underline{n g}=p t$

7. Ribeiro AL, Duncan BB, Brant LCC, Lotufo PA, Mill JG, Barreto SM. Cardiovascular health in Brazil: trends and perspectives. Circulation [serial on the internet]. 2016 [cited 2020 May 11];133(4):42233. Available from: https://pubmed.ncbi.nlm.nih. gov/26811272/

8. Souza MFMD, Malta DC, França EB, Barreto ML. Changes in health and disease in Brazil and its states in the 30 years since the Unified Healthcare System (SUS) was created. Ciênc Saúde Coletiva [serial on the internet]. 2018 [cited 2020 May 11];23(6):1737-50. Available from: https://www. scielo.br/scielo.php?script=sci arttext\&pid $=$ S1413$81232018000601737 \& \operatorname{lng}=$ en \& tlng $=$ en

9. Guimarães RM, Andrade SSCA, Machado EL, Bahia CA, Oliveira MM, Jacques FVL. Diferenças regionais na transição da mortalidade por doenças cardiovasculares no Brasil, 1980 a 2012. Rev Panam Salud Pública [serial on the internet]. 2015 [cited 2020 May 11];37(2):83-9. Available from: https:// scielosp.org/pdf/rpsp/2015.v37n2/83-89/pt

10. Sociedade Brasileira de Cardiologia. Cardiômetro: mortes por doenças cardiovasculares no Brasil [document on the internet]. c2015 [cited 2020 May 10]. Available from: http://www.cardiometro.com. br/default.asp

11. Silva EBF, Silva AL, Santos A0, Dall'Acqua DSV, Souza LFB. Infecções respiratórias de importância clínica: uma revisão sistemática. Revista FIMCA [serial on the internet]. 2017 [cited 2020 May 10];4(1):7-16. Available from: $\quad$ https://www.arca.fiocruz.br/bitstream/ icict $/ 33445 / 2 /$ Infec $\%$ C 3\% A 7\%C 3\%B 5 es $\% 20$ Respirat \% C $3 \%$ B 3 ria s \% 20 d e \% 20 import \%C $3 \%$ A2ncia $\% 20$ cl \%C 3\%ADnica $\% 20$ uma $\% 20$ revis $\%$ C $3 \%$ A 30\%20siatem $\%$ C $3 \%$ A 1 tica.pdf

12. Bárbara C, Melo EG, Nogueira PJ, Farinha CS, Oliveira AL, Alves MI, et al. Portugal: doenças respiratórias em números: 2015. Lisboa: DireçãoGeral da Saúde; 2016.

13. Brasil. Saúde Brasil 2013: uma análise da situação de saúde e das doenças transmissiveis relacionadas à pobreza. 10. ed. Brasília (DF): Ministério da Saúde; 2014.
14. Brasil. Resolução n. 466, de 12 de dezembro de 2012 [document on the internet]. Brasília (DF): Ministério da Saúde; 2012 [cited 2017 Apr 16]. Available from: http://conselho.saude.gov.br/ resolucoes/2012/Res0466.pdf

15. Malta DC, Moura L, Prado RR, Escalante JC, Schmidt MI, Duncan BB. Mortalidade por doenças crônicas não transmissíveis no Brasil e suas regiões, 2000 a 2011. Epidemiol Serv Saúde [serial on the internet]. 2014 [cited 2020 May 10];23(4):599-608. Available from: https://www.scielo.br/pdf/ress/ v23n4/2237-9622-ress-23-04-00599.pdf

16. Malta DC, Oliveira TP, Santos, MAS, Andrade SSCDA, Silva MMAD. Progress with the strategic action plan for tackling chronic non-communicable diseases in Brazil, 2011-2015. Epidemiol Serv Saúde [serial on the internet]. 2016 [cited 2020 May 10];(25):37390. Available from: https://doi.org/10.5123/s167949742016000200016

17. Sociedade Brasileira de Cardiologia. Atlas Corações do Brasil: volume 1 [document on the internet]. 2005 [cited 2020 Mar 30]. Available from: https://www.prefeitura.sp.gov.br/cidade/ secretarias/upload/saude/arquivos/programas/ Atlas CoracoesBrasil.pdf

18.0mran AR. The epidemiologic transition: a theory of the epidemiology of population change. Milbank $Q$ [serial on the internet]. 2005 [cited 2020 Mar 30];83(4):731-57. Available from: https://www.ncbi.nlm.nih.gov/pmc/articles/ PMC2690264/

19. Olshaqnsky SJ, Ault BA. The fourth stage of the epidemiologic transition: The age of delayed degenerative diseases. Milbank $Q$ [serial on the internet]. 1986 [cited 2020 Mar 30]; 64(3):35591. Available from: https://pubmed.ncbi.nlm.nih. gov/3762504/

20. Brasil. Inquérito domiciliar sobre comportamentos de risco e morbidade referida de doenças e agravos não transmissiveis: Brasil, 15 capitais e Distrito Federal, 2002-2003. Rio de Janeiro: Instituto Nacional de Câncer; 2004.

21. Malta DC, Silva Júnior JB. 0 Plano de Ações Estratégicas para o Enfrentamento das Doenças Crônicas não Transmissíveis no Brasil e a definição das metas globais para o enfrentamento dessas doenças até 2025: uma revisão. Epidemiol Serv Saúde [serial on the internet]. 2013 [cited 2020 Mar 30];22(1):151-64. Available from: http://scielo.iec. gov.br/pdf/ess/v22n1/v22n1a16.pdf

22. Oliveira VS, Maia PCGGS, Santos J, Sousa MNA. Análise dos fatores de risco para doenças crônicas não transmissíveis: estudo com colaboradores de uma instituição privada. Saúde (Santa Maria) [serial on the internet]. 2017 [cited 2020 Mar 30];43(1):21424. Available from: https://periodicos.ufsm.br/ revistasaude/article/view/23784 
23. Malta DC, Morais Neto OL, Silva Junior JB. Apresentação do plano de ações estratégicas para o enfrentamento das doenças crônicas não transmissíveis no Brasil, 2011 a 2022. Epidemiol Serv Saúde [serial on the internet]. 2011 [cited 2020 Mar 30];20(4):425-38. Available from: http://scielo. iec.gov.br/pdf/ess/v20n4/v20n4a02.pdf

24. Bacurau AGDM, Francisco PMSB. Prevalência de vacinação contra gripe nas populações adulta e idosa com doença respiratória pulmonar crônica. Cad Saúde Pública [serial on the internet]. 2018 [cited 2020 Mar 30];(34):e00194717. Available from: http://cadernos.ensp.fiocruz.br/csp/artigo/439/ prevalncia-de-vacinao-contra-gripe-nas-populaesadulta-e-idosa-com-doena-respiratria-pulmonarcrnica
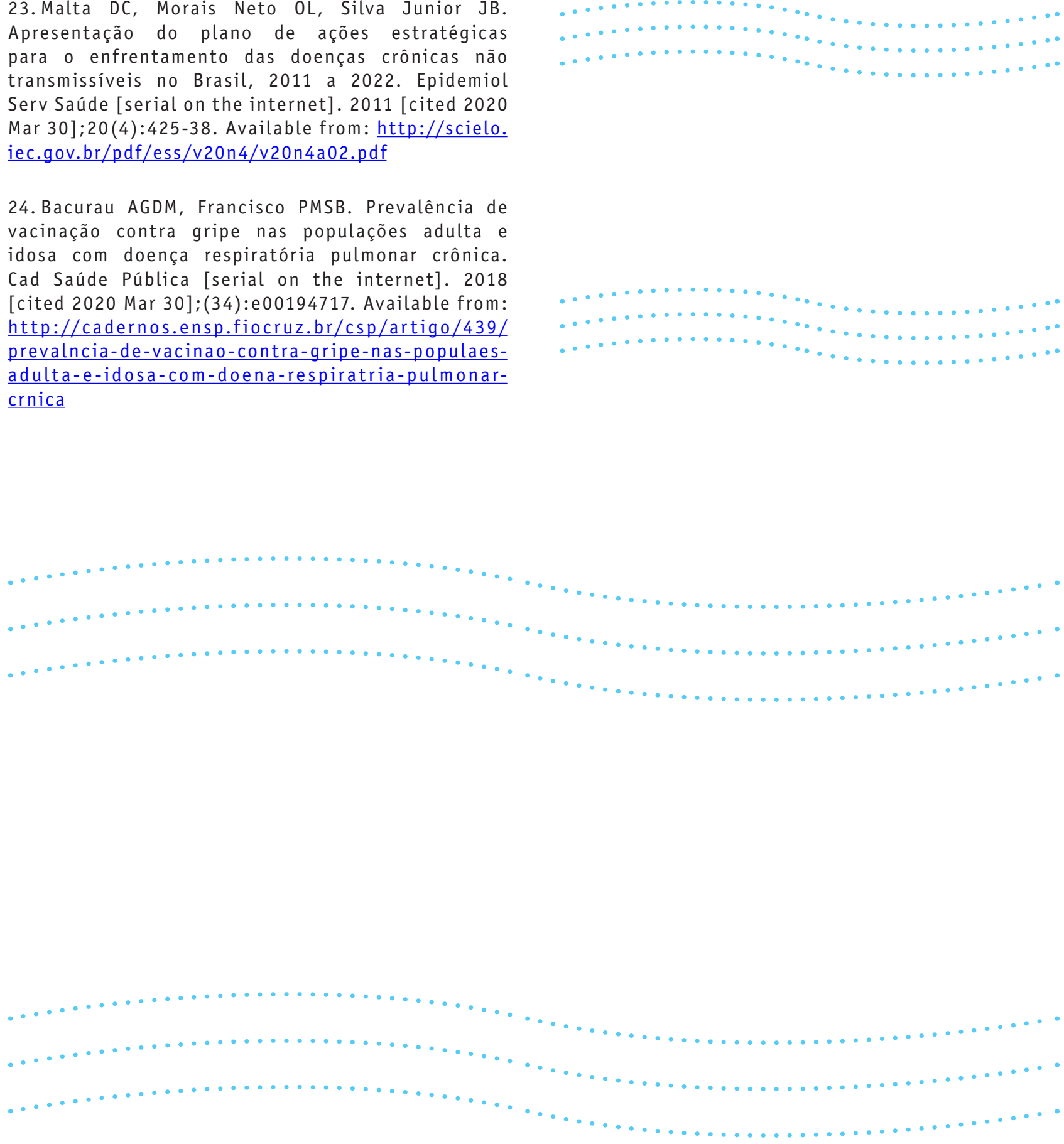